\title{
marketplace
}

\section{Complementary products}

If your patients are prescribed achlorhexidine-basedmouthwash you should recommend that they use a toothpaste free from sodium lauryl sulphate. This is because the sodium lauryl sulphate cancels out the effects of the chlorhexidine.

Curasept'M from Curaprox UK has an improved taste and does not cause staining,

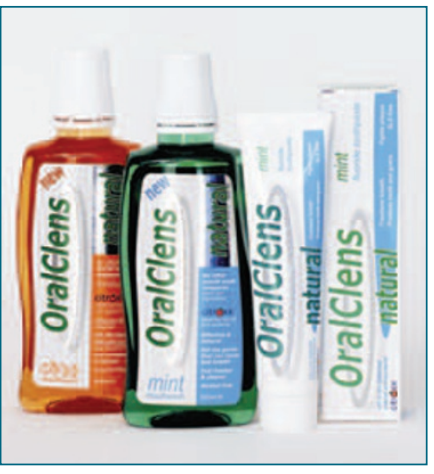

unlike conventional chlorhexidine-based products. It is available as a rinse and a gel.

In complement, Oraldent manufacture Oralclens ${ }^{\mathrm{TM}}$ toothpaste, which is free from sodium lauryl sulphate and can therefore be used with chlorhexidine-based products. Oralclens ${ }^{\mathrm{TM}}$ contains Citroxx ${ }^{\mathrm{TM}}$, a natural mixture of fruit acids and non-toxic bioflavinoids. Citroxx ${ }^{\mathrm{TM}}$ has a clinically proven anti-bacterial action. OralclensTM is also triclosan-free and does not contain CPC, artificial flavourings or sweeteners.

Oralclens ${ }^{\mathrm{TM}}$ is available as an alcohol-free mouthwash as well, for those patients who do not require a chlorhexidine-based product. It is available in citrus or mint flavours.

For further information email sales@oraldent.co.uk, or call 01480862080.

\section{A helping hand}

As all DCPs know, the daily donning of gloves in the dental surgery can make hands dry and in some cases irritated. Maintaining good skincare and providing the ultimate barrier for infections can be a challenge.

METASYS Green \& Clean Professional Hand Care manufactures a range of products for stressed skin: an Antiseptic Hand Wash Lotion, Caring Hand Wash Lotion and Nourishing Hand Cream.

The Hand Wash Lotions contain natural moisturisers and are $\mathrm{pH}$ neutral - particularly useful for frequent hand washing and to protect the natural balance of the skin. The Hand Wash Lotion provides additional infection prophylaxis.

The almond oil-based rich Hand Cream is easily absorbed into the skin and nails without leaving a greasy residue, and is suitable for sensitive skin. Also rich in vitamins E and B it is designed to make your

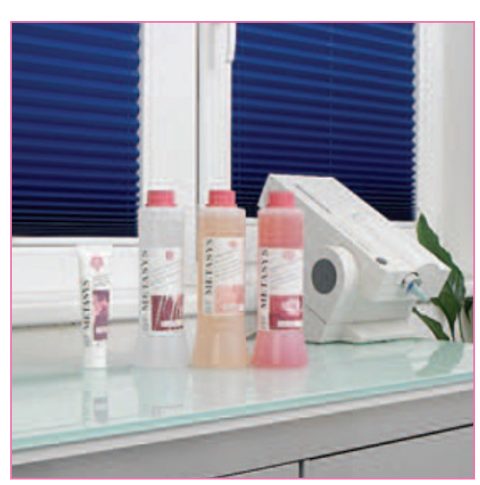
skin smooth and silky soft with regular use, while helping to prevent dehydration.

To complete the range is Green \& Clean HK hand disinfectant, again suitable for sensitive skin and helping maintain an optimum skin balance.

To order METASYS products telephone 08458381636 or visit www.metasys.com.

\section{The wow factor}

What is your patients' first impression when they step into your practice? The interior of your practice can suggest a lot about the quality of care patients can expect to receive from you.

If you want to inject some vibrant colour into your practice, consider Admor's dental art range. Made with fade-proof inks, Admor's reception posters depict radiant, welcoming smiles, colourful toothbrushes and humorous cartoons - created with expert input from dental professionals.

The posters are available in A2 or A3 size, fitted perfectly into purpose-built Snap-shut Wall Frames and acrylic Wall Poster Mounts, all featured in the Admor Interiors Directory.

For the more ambitious, Admor's dental canvas art range are available in long, portrait or square shapes in various sizes. The canvases are digitally printed using water resistant ink, mounted on to deep wooden frames and hand finished.

Admor reception art can be personalised with a caption, logo or the practice name. For further information telephone Admor on 01243 553 078, email print@admor.co.uk or seewww.admor.co.uk.

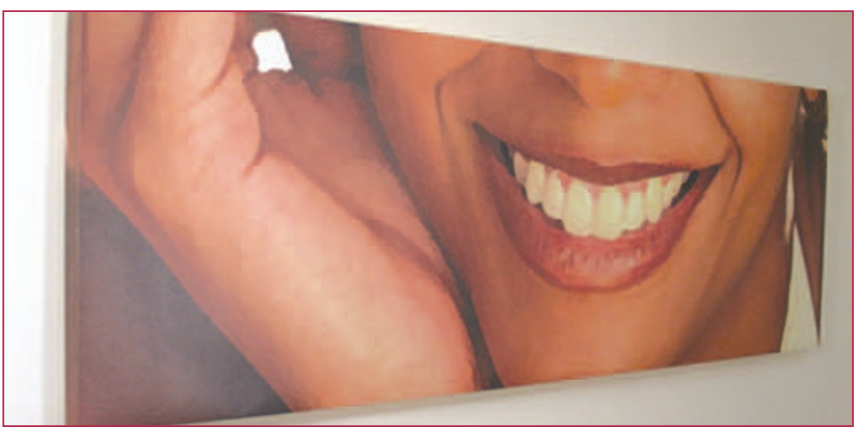

\section{Marketing from within}

Make the most of your existing patients; they can be ambassadors for your practice if you fine-tune your internal marketing.

Effective internal marketing starts with branding and is carried through to brochures, welcome packs, dental menus, referral cards, treatment leaflets, treatment posters and newsletters - all vehicles to educate patients on your full range

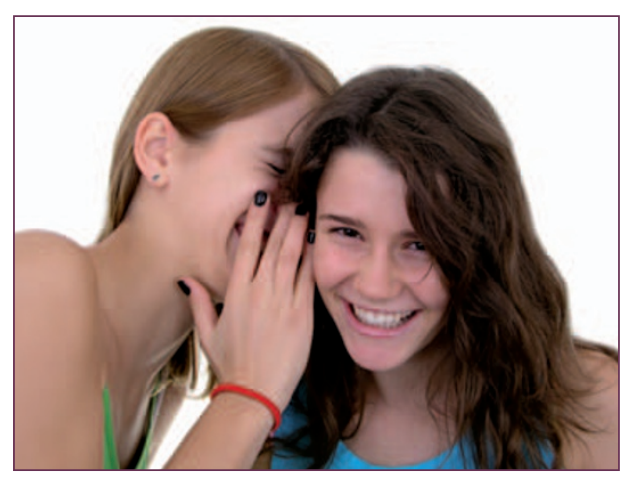
of services and sell more treatments.

Rather than wait for patients to refer other prospective patients to you, referral cards actually request a referral. If patients don't know what you sell then they can't buy.

Designer Dental offers a full internal and external marketing service for dental practices of all sizes, whether NHS or private. Visit www.designerdental.co.uk or telephone 01642206106. 